\title{
Bilateral Brown syndrome in one pair of dizygotic twins: a case
} report

\author{
Abbas Attarzadeh, Abbas Hoseinirad* and Feisal Rahat
}

Address: Poostchi eye research center, shiraz university of medical sciences, shiraz, Iran

Email: Abbas Attarzadeh - attarzaa@sums.ac.ir; Abbas Hoseinirad* - ahrad2@yahoo.com; Feisal Rahat - feisalrahat@gmail.com

* Corresponding author

Published: 2 January 2010

Cases Journal 2010, 3:1 doi:10.1186/1757-1626-3-1

This article is available from: http://www.casesjournal.com/content/3/I/I

(C) 2010 Attarzadeh et al; licensee BioMed Central Ltd.

This is an Open Access article distributed under the terms of the Creative Commons Attribution License (http://creativecommons.org/licenses/by/2.0), which permits unrestricted use, distribution, and reproduction in any medium, provided the original work is properly cited.
Received: 4 December 2008

Accepted: 2 January 2010

\begin{abstract}
Introduction: Brown syndrome is a rare ocular movement abnormality. This syndrome is characterized by an inability to elevate the affected eye in adduction. Most cases are sporadic but the occurrence in Monozygotic twins has suggested the possible autosomal dominant inheritance in Brown syndrome.
\end{abstract}

Case presentation: A 4-year-old girl (one pair of dizygotic twins) was referred to our pediatric ophthalmology clinic to assess her abnormal eye movement noticed by her mother. Visual acuity of both eyes was 20/20 with Snellen chart. Ocular motility showed mild exotropia in primary position with marked divergence in upward gaze ( $\vee$ pattern), mild hypotropia in adduction, and limitation of elevation in adduction of both eyes (Fig. IA). We also examined her sister, all ocular evaluations including visual acuity, slit lamp examination, funduscopy and ocular motility (Ductions \& Versions) were normal without any limitation.

We also review the related articles that previously have reported Brown syndrome in twins.

Conclusion: Although there are few case reports of Brown syndrome in twins, combination of these reports may elucidate the genetic basis of this disease.

\section{Introduction}

In 1950 Brown described an eye movement anomaly as the superior oblique (SO) tendon sheath syndrome [1]. He suspected the existence of a congenitally short SO tendon sheath, which restricted passive elevation of the adducted eye [2]. Brown syndrome is a rare ocular movement abnormality and most cases are sporadic and unilateral, but may be bilateral in $10 \%$ of cases [3]. Familial occurrence has been reported by several authors [4]. Also there are few case reports of Brown syndrome in twins that might be indicative of a possible autosomal dominant or recessive inheritance. Several monozygotic twins with
Brown syndrome have been reported, but we found only two reports of this syndrome in dizygotic twins[4,5]. Here we found bilateral Brown syndrome only in one pair of dizygotic twins.

\section{Case Report}

A 4-year-old girl (one pair of dizygotic twins) was referred to our pediatric ophthalmology clinic to assess her abnormal eye movement noticed by her mother. The patient had a history of 35 weeks gestational age at birth time and her birth weight was 1700 grams. Parents did not report any history of congenital abnormalities in the family. 
Pregnancy history was negative for exposure to drugs or teratogens. Visual acuity of both eyes was 20/20 with Snellen chart. Assessment of stereo acuity using the Titmus test wasn't possible. Cycloplegic refraction was $+0.25 \times 90^{\circ}$ for both eyes. Ocular motility showed mild exotropia in primary position with marked divergence in upward gaze (V pattern), mild hypotropia in adduction, and limitation of elevation in adduction of both eyes (Fig. 1A). The patient had mild lid retraction in the primary position, but laboratory examination and consultation with relevant specialists was within normal limits. Fundus examination of both eyes was normal without any sign of objective torsion. All other examinations were within normal limits. We also examined her sister, all ocular evaluations including visual acuity, slit lamp examination, funduscopy and ocular motility (Ductions \& Versions) were normal without any limitation. The past medical history of this pair was 1920 grams birth weight without any other problem.

\section{Discussion}

Brown syndrome is caused by a mechanical restriction of the superior oblique tendon moving through the trochlea, which results in a restriction of elevation in adduction. Most cases of Brown syndrome are congenital, but it can be acquired due to either trauma or inflammation.

The occurrence of Brown syndrome, either bilateral or unilateral in one or both pairs of twins has been reported by several authors[4-9]. From these reports, only Magli et al. and Caldeira have reported ocular movement abnormality compatible with Brown syndrome in dizygotic twins, and other reports from Katz et al., Finaly et al., Lowe et al and kim et al. have been in monozygotic twins $[5-7,9]$. Table 1 summarizes these findings. Brown syndrome in reports of Finaly et al., Katz et al., kim et al. was equal in both pairs but Lowe $\mathrm{RF}^{8}$ and Magli et al have found the Brown syndrome only in one pair of monozygotic and dizygotic twins, respectively[8]. In our report, we found that only one pair of dizygotic twins was affected and ocular motility of the other pair was without problem.

Although all of the previous reports support a possible autosomal dominant or recessive inheritance with incomplete penetrance and variable expression, our case presentation like reports of Magli et al. and Caldeira strengthens the possibility of autosomal dominant with incomplete penetration or autosomal recessive transmission, because this syndrome was found only in one pair of twins and search for finding of similar disease in their siblings was negative[2].

Brown syndrome is a rare ocular motility abnormality and it seems if we merge these scattered case reports in twins, probable genetic basis as one of the ethiologic factors in Brown syndrome will be highlighted.
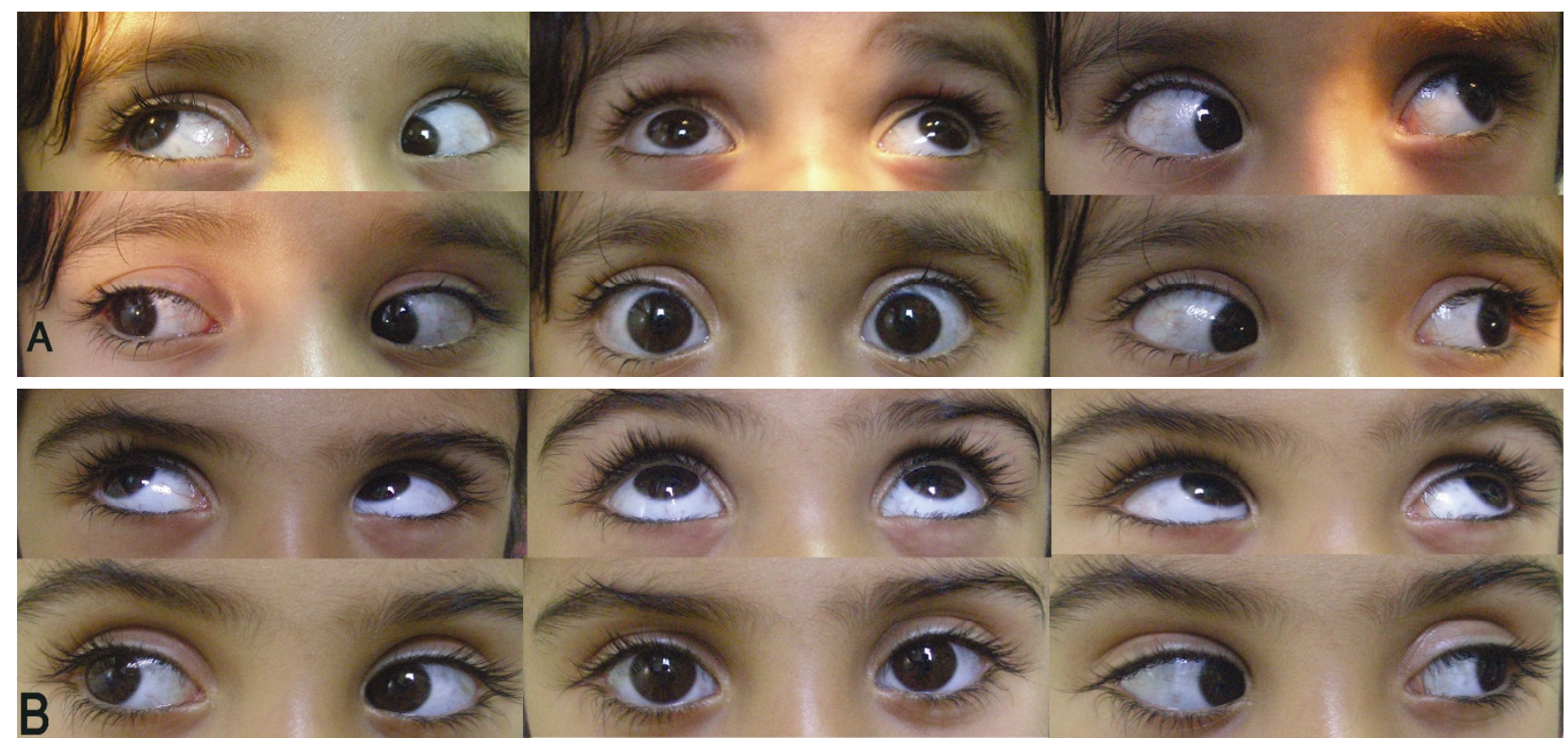

\section{Figure I}

A). Ocular movements of affected pair; note limitation of elevation in adduction of both eyes. B) Normal eye motility of the other pair. 
Table I: Reported cases of brown syndrome in twins

\begin{tabular}{llll}
\hline Report & Affected Individuals & Gender & Laterality \\
\hline Lowe RF & Monozygotic & F & Bilateral \\
Magli et al & Dizygotic & M & Unilateral \\
Katz et al & Monozygotic & F & Unilateral \\
Finlay et al & Monozygotic & F & Unilateral \\
Kim et al & Monozygotic & F & Bilateral \\
Our report & Dizygotic & F & Bilateral
\end{tabular}

According to our search in the literature (MEDLINE from 1950 until 2008) we didnot find similar case report (bilateral Brown syndrome in one pair of dizygotic twins).

Our key words for search in MEDLINE were Brown syndrome with bilateral and twins.

Written informed consent was obtained from the patient for publication of this case report and accompanying images. A copy of the written consent is available for review by the Editor-in-Chief of this journal.

\section{Competing interests}

The authors declare that they have no competing interests.

\section{Authors' contributions}

AA participated in its design and coordination. AH wrote the article and provided the relevant material. FR done internet search and provided the patient photos.

All authors read and approved the final manuscript.

\section{References}

I. Brown HW: Congenital structural muscle anomalies. In Strabismus Ophthalmic Symposium Edited by: Allen JH. St. Louis: CV Mosby; 1950:205-36

2. Weakley DR, Stager DR, Stager DR: "Brown syndrome". In Clinical Strabismus Management Edited by: Rosenbaum AL, Santiago AP. Phliadelphia: WB Saunders; 1999:347-57.

3. Wilson MM, Eustis HS Jr, Parks MM: Brown's syndrome. Surv Ophthalmol 1989, 34:153-72.

4. Caldeira JA: Presumptive Brown's syndrome in dizygotic female twins: Case report and review of $\mathbf{3 0}$ familial cases in the literature. Binocular Vision Strabismus Q 1996, I I:23.

5. Katz NK, Whitmore PV, Beauchamp GR: Brown's syndrome in twins. J Pediatr Ophthalmol Strabismus 1981, 18:32-4.

6. Magli A, Fusco R, Chiosi E, Del Bono G: Inheritance of Brown's syndrome. Ophthalmologica 1986, 192:82-7.

7. Finlay A, Powell S: Brown's syndrome in identical twins. $\mathrm{Br}$ Orthop J 1982, 39:73-7.

8. Lowe RF: Bilateral superior oblique tendon sheath syndrome occurrence and spontaneous recovery in one of uniocular twin. Br J Ophthalmol 1969, 53:466-7I.

9. Kim SH, Ben-Zion I, Neely DE: Bilateral Brown syndrome in monozygotic twins. J AAPOS 2008, 12:193-194.

10. Von Noorden GK, Campos EC: Binocular Vision and Ocular Motility. 6th edition. St. Louis, Mosby; 2002:466-71.

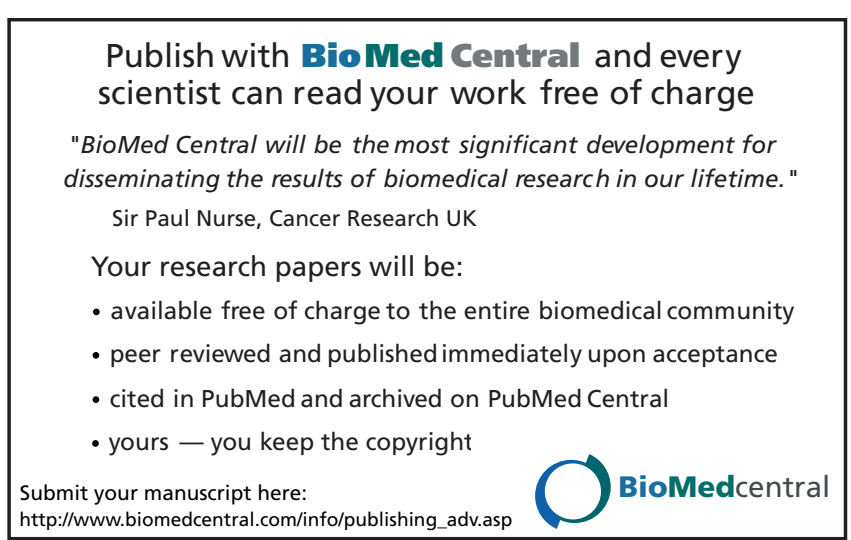

